\title{
X-ray triggered PD measurements in small sized spherical voids at the detection limit
}

\section{Conference Paper}

Author(s):

Schmitt, Cédric; Hermann, Lorenz G.; Pancheshnyi, Sergey; Adili, Sedat; Franck, Christian (D)

Publication date:

2014

Permanent link:

https://doi.org/10.3929/ethz-b-000090907

Rights / license:

In Copyright - Non-Commercial Use Permitted

Originally published in:

https://doi.org/10.1109/CEIDP.2014.6995903 


\section{(C) 2014 IEEE.}

Personal use of this material is permitted. Permission from IEEE must be obtained for all other uses, in any current or future media, including reprinting/republishing this material for advertising or promotional purposes, creating new collective works, for resale or redistribution to servers or lists, or reuse of any copyrighted component of this work in other works."

Digital Object Identifier: 10.1109/CEIDP.2014.6995903 


\title{
$\mathrm{X}$-ray triggered PD measurements in small sized spherical voids at the detection limit
}

\author{
C. Schmitt ${ }^{1}$, L.G. Herrmann ${ }^{1}$, S. Pancheshnyi ${ }^{1}$, S. Adili ${ }^{2}$, C.M. Franck ${ }^{2}$ \\ ${ }^{1}$ ABB Switzerland Ltd, Corporate Research, CH-5405 Baden-Dättwil, Switzerland \\ ${ }^{2}$ Power Systems and High Voltage Laboratories, ETH Zurich, 8092 Zurich, Switzerland \\ Lorenz.Herrmann@ch.abb.com
}

\begin{abstract}
Pulsed X-ray triggered PD measurements were introduced in electrical testing in order to eliminate the statistical time lag. Moreover it was shown recently that the PD mechanism in air filled voids in epoxy insulation does not change with respect to natural inception of PD. In this paper we address the question of the detection limit of this method concerning the void size. In the case of bisphenol A based epoxy, as used in real GIS spacer insulators, we find clear indication that air-filled voids can be reliably triggered to diameters down to $0.3 \mathrm{~mm}$. Below this limit, the deposited charge becomes small and approaches the detection limit of the PD system. Therefore we conclude that our method is not limited by the size of the void.
\end{abstract}

Keywords-partical discharge measurments; voids; detection limit; epoxy insulation; X-ray triggered PD.

\section{INTRODUCTION}

Conventional PD measurements in solid insulators are subject to a time lag between voltage application and PD inception [12]. The reason is that inception only takes place as soon as a starting electron is available which is a statistical effect and can take days for small void sizes $<0.5 \mathrm{~mm}$ [3]. For industrial applications like factory outgoing tests, however, testing time has to be reduced to a minimum and therefore immediate inception after voltage application is highly desirable [4]. In order to overcome the time lag, X-rays were suggested to supply starting electrons artificially in the moment of voltage application [5-6]. As continuous X-rays can have negative impact on PD mechanism and measurements, ultrashort X-ray pulses were found to overcome the limits of continuous X-rays [7-8]. Pulses are applied at maximum AC voltage but due to their duration of only some nanoseconds they do not disturb the phase resolved PD pattern of the void [9]. In order to show that the Pulsed X-ray Induced Partial Discharge (PXIPD) technique leads to the same PD mechanism than naturally incepted discharges, time-resolved current and light emission measurements were made which showed that naturally and Xray triggered pulses have identical shapes [10].

In this paper we address the question of the minimal void size which can be detected with PXIPD. In the past [8], samples including small voids showed a higher inception field than expected by current theory [3]. By further developing our fabrication method and theoretical understanding we can show that for samples made of the epoxy base material used in real GIS spacers we can reliably trigger voids down to the noise level of the electronic detection circuit.

\section{EXPERIMENTAL}

\section{A. PD measurement circuit}

As soon as the applied AC voltage reaches a value above the predicted inception level of the void [3] a trigger signal enables the X-ray source which generates a pulse of 30-50 ns duration. The measurement circuit including an example void embedded between two electrodes is illustrated in Figure 1.

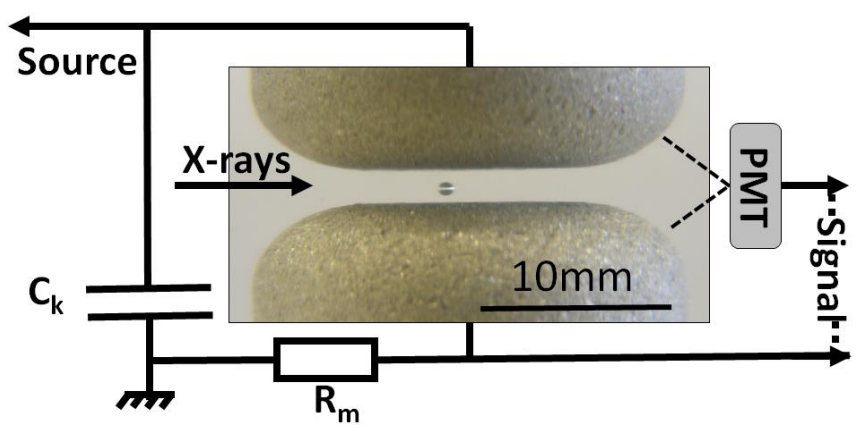

Fig. 1. PD detection circuit with sample including artificial void.

With our setup we can measure optical and electrical PD signal simultaneously and time resolved. In order to measure short pulses we use a digitizing oscilloscope with a bandwidth of $1 \mathrm{GHz}$ with $10 \mathrm{GS} / \mathrm{s}$. Also the circuit has to be adjusted to the time resolved measurements: In order to achieve high bandwidth, a low stray inductance and capacitance is necessary. This is achieved by designing the ground and highvoltage electrode in a way that they can be used directly as coupling capacitor with $\mathrm{C}_{\mathrm{k}}=61 \mathrm{pF}$ [10]. The PD current transformed into a voltage signal with $\mathrm{R}_{\mathrm{m}}=50 \Omega$ is transmitted by a $50 \Omega$ transmission line. The optical signal is detected by a PMT module (Hamamatsu H10721-110) with built- in power supply and spectral response from 230nm to 700nm [10]. Alternatively, the electrical signal can be measured phaseresolved and can be presented in the standard diagrams [3], where charge magnitude is shown as a function of the phase of the applied voltage. Detection sensitivity is $<1 \mathrm{pC}$. 


\section{B. Sample Fabrication}

We fabricate our samples in a two-step process: First we produce small epoxy blocks with edge lengths smaller than $2 \mathrm{~mm}$ containing one isolated void with diameters between $0.3 \mathrm{~mm}$ and $1.5 \mathrm{~mm}$. To produce these blocks we inject air bubbles into epoxy at the moment of gelation. Due to the increased viscosity air bubbles do not rise but get stuck in the hardening epoxy. As this is a statistic process we get a distribution of arbitrary bubble sizes. After curing the material we cut out blocks one by one and measure the diameter with an optical microscope. Second we embed the small blocks between two cylindrical electrodes with distance $3 \mathrm{~mm}$ within an additional epoxy casting step and obtain a homogenous sample with a single, spherical void between the electrodes. The material used for the small block will henceforth be labelled block material whereas the surrounding material used for the second casting step is labelled matrix material.

We fabricate two different sample types. For type one (subsequently labeled GIS) both block and matrix material are made of the same epoxy as used in ABB GIS spacer insulators. For type 2 (labeled DM) we use the model material system introduced previously [7-8]. The block is made of a special room temperature curing epoxy while cycloaliphatic epoxy is used for the bulk. Hence in this type, block and bulk are made of different materials.

\section{Delamination of inserted blocks}

The interface between the incorporated block including the void and the surrounding matrix material is critical. Especially if inception voltages of the voids are to be measured we have to make sure that delamination noise coming from the interface is not mistaken for PD inception of the void. A means to distinguish between the two phenomena, i.e. delamination noise and regular PD signal coming from the void, is to study the phase resolved PD pattern.

In Figure 2 we present phase resolved PD patterns for a single half-wave of the $50 \mathrm{~Hz}$ excitation voltage above the inception thresholds of the samples. In Figure 2(a) we present a PD pattern of a sample in which matrix material and inserted block are made of different materials. The patterns of such voids often differ from patterns expected for spherical voids. As delamination between electrode and epoxy is the most likely source of the additional features in the signal, the pattern is considered to suffer from delamination noise. For rather big voids with diameters of $1 \mathrm{~mm}$ or larger this does not play a role because the expected charge coming from the PD in the void is much bigger. Hence PD inception in the void can be reliably measured. Small voids $(d=0.4 \mathrm{~mm})$ as shown in the Figure, however, lead to PD charges of more or less the same magnitude as the charge coming from the delamination. Hence it is difficult to distinguish noise and PD coming from the void in a reliable way. To eliminate the delamination noise we change two things: First we manufacture the incorporated block of the same material as the surrounding matrix material and second we refine the casting process by gluing the block to the top electrode with a drop of liquid epoxy before we start filling the mould. With this method a fraction of more than $60 \%$ of the produced samples are delamination free and lead to clean phase resolved PD patterns as described in reference [3] and illustrated in Figure 2(b). The void diameter of the sample shown here is $d=0.4 \mathrm{~mm}$. To prove the concept we also cast several reference samples which include a void-free block and incorporate the block between the two electrodes of our sample. As can be seen in Figure 2(c) the phase-resolved pattern does not show any PD. Hence we clearly show that we can produce delamination-free samples with our method.

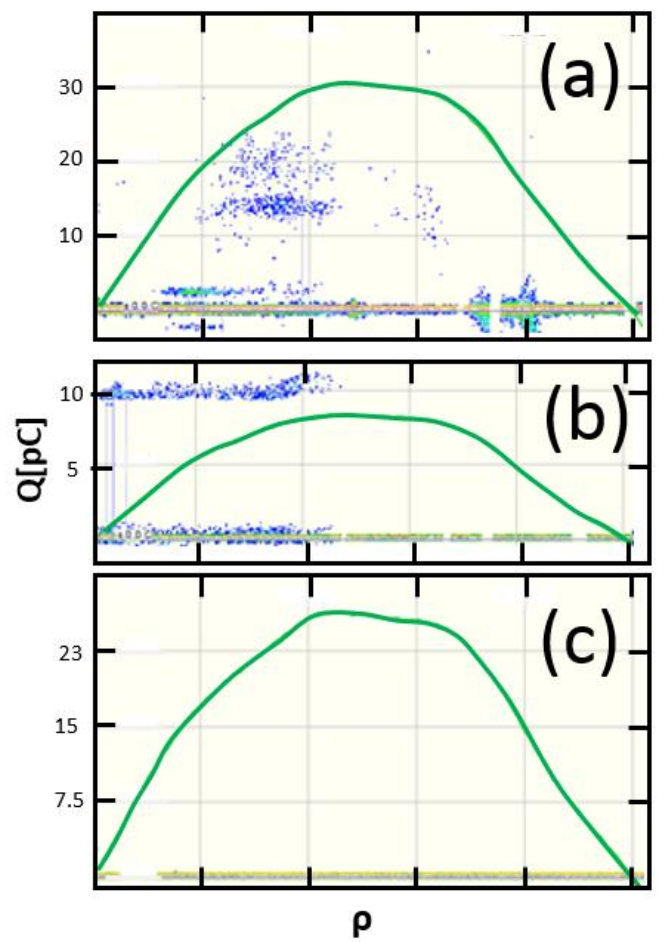

Fig. 2. Phase-resolved PD patterns for different situations: (a) void size $0.43 \mathrm{~mm}$, block and matrix are of different materials (b) void size $0.41 \mathrm{~mm}$, block and matrix are made of the same material (c) reference block without void, made of the same material as the matrix.

\section{RESULTS AND DISCUSSION}

We use the paper by Gutfleisch and Niemeyer [3] to compare our experimental data to model predictions. We choose two parameters for this comparison namely inception voltage and measured PD charge as a function of void size.

\section{A. Inception}

The inception field $E_{s t r}$ of streamer discharges in spherical voids is given by

$$
f E_{0}>E_{s t r}=(E / p)_{\mathrm{cr}} \mathrm{p}\left[1+\frac{\mathrm{B}}{(2 \mathrm{pR})^{n}}\right]
$$

The factor $f$ quantifies the field enhancement in the void and is $\sim 1.33$ for spherical voids in epoxy with $\varepsilon_{r}=4$. E0 is the applied background field and $(E / p)_{c r i t}=25 \mathrm{~V} /(\mathrm{mPa}), B=8.6 \mathrm{~m}^{1 / 2} \mathrm{~Pa}^{1 / 2}$ and $n=0.5$ are gas constants for air. The pressure $p$ in the void is typically assumed to be in the range of $p=0.1-1$ bar. The result which we obtained for Pulsed X-ray triggered inception is illustrated in Figure 3. All 11 samples with diameters down to $0.3 \mathrm{~mm}$ in which block and bulk are both made of GIS epoxy 
incept as predicted by equation (1) assuming pressures between 0.4 and 1 bar. All GIS samples presented here are delamination free. For each sample multiple PXIPD measurements are made: We start with an excitation voltage of $1 \mathrm{kV}$ and then increase the voltage in $1 \mathrm{kV}$ steps. The voltage of first PD inception is used to calculate the inception field of the sample.

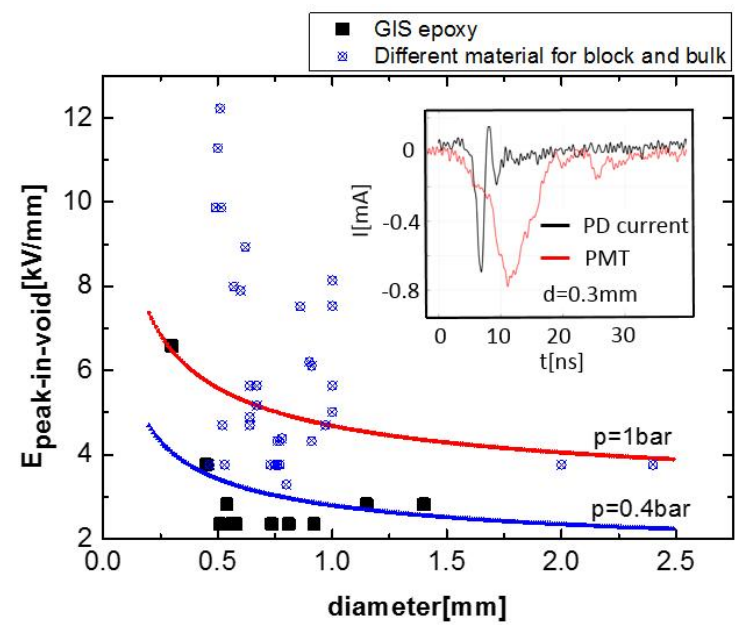

Fig. 3. PD Inception fields for GIS epoxy samples and samples as used previously [8] with different materials for block and bulk. The inset shows the time-resolved signal for a very small GIS sample with $\mathrm{d}=0.3 \mathrm{~mm}$.

Partial discharge inception is visible both in the phase resolved measurement (see Figure 2) as well as within a time-resolved measurement as can be seen in the inset of Figure 3. The time resolved signal is obtained electrically and confirmed by an optical PD signal. The shape of the time resolved signal is qualitatively equal to the signal recorded for larger voids [10]. Hence we obtain indications that we indeed observe PD as assumed. In contrast to GIS material, PD inception of DM samples with diameters $<1 \mathrm{~mm}$ cannot be described by equation (1). Indeed about half of the samples incepted at higher fields than predicted. Moreover more than half of the samples showed phase-resolved PD patterns typical for voids plus delamination of the blocks (see Figure 2). Therefore some of the points could in principle also be due to delamination noise. We try to minimize the error by comparing the phase resolved pattern to typical void like patterns and take into account 35 samples in order to have statistical significance.

\section{B. Deposited Charge}

The different results in inception voltage of different sample types (i.e. DM and GIS samples) as shown in Figure 2 can have two possible reasons: First the discharge mechanism in the two sample types could be different and second there could be a difference in the availability of the starting electron. Concerning the discharge mechanism we first compare the pulse shape of PD pulses obtained from different sample types: All pulses look qualitatively the same as depicted in inset of Figure (3). Hence pulse shape does not give any indication that the PD mechanism for different sample types is different. A second indication of similar PD mechanism in different sample types is given by the measurement of the apparent minimal charge (i.e. the charge detected by the PD system) and the comparison to the theoretical value expected for streamer discharges in spherical voids [12]:

$$
q_{\min }=4 \pi\left(\frac{d}{2}\right)^{3} \varepsilon_{0} \varepsilon_{r} \frac{p}{L}(E / p)_{c r}\left[1-\gamma+\frac{B}{\sqrt{p d}}\right]
$$

Here $d$ is the diameter of the void, $L$ is the electrode distance, $\varepsilon_{0}$ and $\varepsilon_{r}$ are vacuum and dielectric permittivity, respectively, $p$ is the pressure in the void, $\gamma \sim 0.35$ is the ratio between residual and critical field for air.

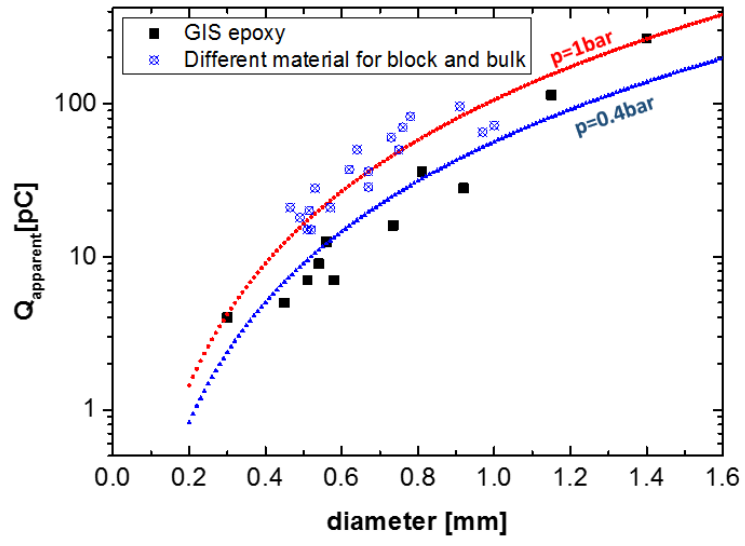

Fig. 4. Apparent minimal charge measured as a function of void size. Both data for GIS epoxy samples and DM samples are compared to expected charge values derived by the streamer criterion [12].

As depicted in Figure 4, measured charge values are well explained by the streamer criterion assuming pressures between 0.4 and 1 bar. Note that this is the case for both GIS and DM samples. Hence we do not observe a substantial difference between the discharges, once started, within the two sample types. Together with the similarities concerning the pulse shapes this result gives a strong indication that discharge types are the same in both sample types. In the following we discuss two hypothesis which could explain the different inception behaviors. Note that experimental evidence for neither of the two is available at the moment.

\section{Material dependence of PD inception}

Our result indicates that PD inception in air-filled voids with diameters $<0.8 \mathrm{~mm}$ depends on the surrounding material.

A first scenario to explain this is that a very first discharge is triggered but remains undetected by our time-resolved system, and no subsequent PDs occur. The first discharge deposits charges on the void surface, which typically act as a source of first electrons for subsequent PD discharges. It is known that the probability for a next first electron release depends on the properties of the void surface [17] and that charges decay if the surface conductivity is too high. A typical time-scale for charge decay is given in [17] by:

$$
\tau \sim \varepsilon_{0} d /(2 \kappa)
$$

where $\varepsilon_{0}$ is the vacuum permittivity, $d$ the diameter of the void and $\kappa$ the surface conductivity. For PD activity to be stopped after the first discharge, $\tau$ must be smaller than one half-wave of the $50 \mathrm{~Hz}$ excitation voltage. Hence the required 
surface conductivity for a void with $d=0.8 \mathrm{~mm}$ to become an equipotential sphere within $\tau=10 \mathrm{~ms}$ would be $\kappa \sim 10^{-13} \Omega^{-1}$ which, according to reference [18], is a high but still reasonable value for the surface conductivity of epoxy. Hence it is possible that in the case of DM samples the surface conductivity is higher than this threshold, whereas it is lower in the case of GIS samples.

A second attempt to explain our observation is the hypothesis that a start electron is always available in the case of GIS samples, whereas this is only sometimes the case in DM samples. Therefore we reconsider the availability of the starting electron in air-filled spherical voids as a function of void size and deposited dose. For real High Voltage components and big spherical voids with diameters of roughly $1 \mathrm{~mm}$ this topic has been studied by Adili et al. [13]. As presented elsewhere [14] in more detail we extend this study to small voids $<0.8 \mathrm{~mm}$. In this regime the influence of the photon energy on the interaction between radiation and gas molecules has to be taken into account. The total probability $P$ of interaction between photon flux $\varphi$ and the gas in the void with density $\rho$ and mass attenuation coefficient $\mu$ within the void volume $V$ can be estimated by $P=\varphi \mu \rho V$. For oxygen and nitrogen we obtain $\rho=1.2 \times 10^{-3} \mathrm{~g} / \mathrm{cm}^{3}$ and $\mu=0.15 \pm 0.05$ $\mathrm{cm}^{2} / \mathrm{g}$ for photons with energies $30-300 \mathrm{keV}$ [15]. The radiative dose $D$ with units $[\mathrm{J} / \mathrm{kg}]$ can be calculated from the exposure $\mathrm{X}[\mathrm{C} / \mathrm{kg}]$ as $D=X(W / e)$ where the quantity $W$ with $(\text { W/e })_{\text {air }}=33.97 \mathrm{~J} / \mathrm{C}[16]$ is the mean energy expended in the gas per ion pair formed and $e$ is the elementary charge. For low-atomic number materials and photon energies $E_{p h}$ below $1 \mathrm{MeV}$ the radiative dose can also be expressed by $D=\varphi E_{p h} \mu$. Hence we can equalize the two equations for $D$ to obtain $\varphi=X(W / e) /\left(E_{p h} \mu\right)$. Hence we can write:

$$
P_{\text {air }}=\frac{\pi}{6}\left(\frac{W}{e}\right) \frac{\rho d^{3}}{E_{p h}} X=3.4 \times 10^{10} \frac{(d[\mathrm{~cm}])^{3}}{E_{p h}[\mathrm{eV}]} X[\mathrm{R}]
$$

The energy distribution of the Golden Engineering XRS-3T $\mathrm{X}$-ray source which we use has its maximum at approximately $55 \mathrm{keV}(68 \%)$ and decays towards the $300 \mathrm{keV}$ cut-off [13]. At a distance of $20 \mathrm{~cm}$ from the source we measure an exposure of $\boldsymbol{X}=10 \mathrm{mR}$ per pulse. If we set equation (4) equal to 1 and assume that the used XRS-3T source is placed at a distance of $20 \mathrm{~cm}$ in front of the samples, the gas volume of voids can be ionized down to void diameters of $0.5-0.6 \mathrm{~mm}$. If this is true, the mechanism to produce starting electrons below this threshold changes and the X-ray/epoxy interaction is responsible for first electron production.

Note that both explanations are hypothesis and lack experimental evidence at the moment. Also the numbers presented constitute only a rough estimate. Further research in these two directions is necessary to come to more precise results.

\section{CONCLUSION}

In this paper we have shown that PXIPD triggers artificial air filled voids in material used in real GIS insulators down to diameters of $0.3 \mathrm{~mm}$. For these void sizes expected charges are roughly $1 \mathrm{pC}$, hence close to the noise limit of commercially available PD equipment. Furthermore we have delivered indications that below a size threshold of diameters around $0.8 \mathrm{~mm}$ the mechanism to incept partial discharges is material dependent. Further work is necessary to understand the physical nature of this interaction.

\section{REFERENCES}

[1] S. A. Boggs, "Partial discharge. III. Cavity-induced PD in solid dielectrics", Electrical Insulation Magazine, IEEE, vol. 6, pp. 11-16, 1990.

[2] A. Cavallini, F. Ciani, G. Mazzanti, G.C. Montanari, "First electron availability and partial discharge generation in insulation cavities: effect of light irradiation", Dielectrics and Electrical Insulation, IEEE Transactions on, vol. 12, pp. 387-394, 2005.

[3] F. Gutfleisch and L. Niemeyer, "Measurement and simulation of PD in epoxy voids", Dielectrics and Electrical Insulation, IEEE Transactions on, vol. 2, pp. 729-743, 1995.

[4] D. Tehlar, U. Riechert, G. Behrmann, M. Schraudolph, L.G. Herrmann, S. Pancheshnyi, "Pulsed X-ray induced partial discharge diagnostics for routine testing of solid GIS insulators", Dielectrics and Electrical Insulation, IEEE Transactions on, vol. 20, pp. 2173-2178, 2013.

[5] B. Fruth and L. Niemeyer, "The importance of statistical characteristics of partial discharge data", Electrical Insulation, IEEE Transactions on, vol. 27 , pp. 60-69, 1992

[6] N. Fujimoto, S. Rizzetto, J.M. Braun, "Partial discharge. XV. Improved PD testing of solid dielectrics using X-ray induced discharge initiation", Electrical Insulation Magazine, IEEE, vol. 8, pp. 33-41, 1992.

[7] S. Adili, C.M. Franck, H. Fuhrmann, "Pulsed X-ray Induced Partial Discharge Measurements", Proceedings of the Gas Discharge Conference, 606, 2010.

[8] S. Adili, C.M. Franck, S. B. Sert, L.G. Herrmann, "Pulsed X-ray Induced Partial Discharge: Phase and Time-Resolved Measurements", IEE Ann. Report Conf. El. Ins. and Dielec. Phenomena, Vol. 1, pp. 3235, 2011.

[9] P.H.F. Morshuis, "Partial discharge mechanisms", Technische Universiteit Delft, 2013.

[10] S. Adili, L.G. Herrmann, C.M. Franck, "Investigating the inception mechanism of pulsed X-ray triggered partial discharges by time resolved measurements", Dielectrics and Electrical Insulation, IEEE Transactions on, vol. 20, pp. 1780-1788, 2013.

[11] J. M. Wetzer and P. C. T. Van Der Laan, "Prebreakdown currents: Basic interpretation and time-resolved measurements", Electrical Insulation, IEEE Transactions on, vol. 24, pp. 297-308, 1989.

[12] F. Gutfleisch. L. Niemeyer, "Measurement and Simulation of PD in Epoxy Voids", IEEE Transactions on Dielectrics and Electrical Insulation, 2(5), pp. 729-743, 1995.

[13] S. Adili and C. M. Franck, "Application of pulsed x-ray induced partial discharge measurements", Dielectrics and Electrical Insulation, IEEE Transactions on, vol. 19, pp. 1833-1839, 2012.

[14] U. Riechert, D. Tehlar, G. Behrmann, L.G. Herrmann, S. Pancheshnyi, "Application of Pulsed X-ray Induced Partial Discharge Diagnostics during Routine Testing", CMD 2014, unpublished.

[15] NIST Photon Cross Sections Database (2010), http://www.nist.gov/pml/data/xcom/.

[16] J. Bushberg, "The Essential Physics of Medical Imaging", Lippincott Williams \& Wilkins, 2002.

[17] L. Niemeyer, "A generalized approach to partial discharge modeling", Dielectrics and Electrical Insulation, IEEE Transactions on, vol. 2, pp. 510-528, 1995.

[18] C. Hudon, R.Bartnikas, M.R. Wertheimer, "Spark-to-glow discharge transition due to increased surface conductivity on epoxy resin specimens", Electrical Insulation, IEEE Transactions on, vol. 28, pp. 18,1993 . 